RHIC and the Pursuit of the Quark-Gluon Plasma

Jeffery T. Mitchell

July 25, 2001

Prepared for:

Brookhaven National Laboratory

Under Contract No. DE-AC02-98CH10886 


\title{
RHIC and the Pursuit of the Quark-Gluon Plasma
}

\author{
Jeffery T. Mitchell \\ Brookhaven National Laboratory \\ Upton, NY 11973
}

Mitchell@,bnl.gov

July 25,2001 


\title{
RHIC and the Pursuit of the Quark-Gluon Plasma
}

\author{
by Jeffery T. Mitchell \\ Brookhaven National Laboratory \\ 2001 Sambamurti Lecture Article
}

\section{Introduction}

There is a fugitive on the loose. Its name is Quark-Gluon Plasma, alias the QGP. The QGP is a known informant with knowledge about the fundamental building blocks of nature that we wish to extract. This briefing will outline the status of the pursuit of the elusive QGP. We will cover what makes the QGP tick, its modus operandi, details on how we plan to hunt the fugitive down, and our level of success thus far.

\section{Quark-Gluon Plasma: Dossier}

The best way to understand the QGP is to break down the words in its name, starting with the word quark. The quark is one of the fundamental particles in nature. If you examine an atom, you will see that the atom is composed of a nucleus with electrons orbiting it. If you look closely at the nucleus, you will see that it is made up of protons and neutrons, also referred to as nucleons. If you look inside the nucleons, you will see that they are made up of three quarks each. A quark can be one of six flavors: $u p$, down, strange, charm, top, or bottom. For instance, the proton consists of two up quarks and one down quark, while the neutron contains two down quarks and one up quark. The lightest of the quarks are the up and down quarks, which are the ones we will be dealing with on a regular basis. The strange and charm quarks are heavier and harder to produce, but we will come across these quarks often. The heaviest top and bottom quarks are not involved in our search for the QGP.

The second word in the name is gluon, which is a particle that can also be found within the protons and neutrons of the nucleus. Beware that the QGP is known to use force, and that force is strong. Like all of the fundamental forces, the strong force is transmitted by a particle. For example, the electromagnetic force is transmitted by the photon and the gravitational force is transmitted by the graviton (which has yet to be observed directly). The strong force is transmitted between the quarks by the gluon. Unlike the gravitational and electromagnetic forces, which decrease in force dramatically as the distance between the two interacting objects increases, the strong force will increase dramatically as the separation between two quarks increases. This behavior of the strong force tends to bind quarks together. Quarks are very communal particles and have never been found alone in nature.

The final word in the name is plasma. Quarks are always found bound together in groups of two or three, called hadrons. If a particle contains three quarks, it is further classified as a baryon. The proton and the neutron are examples of baryons. If a particle contains two quarks, it is classified as a meson. Pions and kaons are examples of mesons. Our suspect can be very mischievous when it comes to quarks by creating a volume of matter (the plasma) in which the bindings between the quarks are broken and the quarks are free to roam as individuals - against their nature. We plan to catch the QGP in the act of creating this plasma phase and essentially setting the quarks free.

\section{How to Catch a Plasma}

Here is an outline of our strategy for catching the elusive QGP. Generally, we have a good idea where our suspect likes to hide out. What we plan to do is build an environment where the QGP would be comfortable and stake out the area until it shows itself. Our approach will be to induce a phase transition to the plasma by applying heat and pressure to normal nuclear matter. This process is similar to what is done when changing water to ice. If you add sufficient pressure to liquid water, you can change it to ice. Also, if you remove heat from water, you can also change it to ice. These processes can be 
illustrated in a phase diagram, where the phase of the matter is mapped out as a function of the pressure and temperature of the system, as shown in Figure 1a. A similar phase diagram can be plotted for nuclear matter, as shown in Figure 1b. Here, if we apply enough heat, and/or enough pressure, to normal nuclear matter, we could change it into the Quark-Gluon Plasma phase that we seek. With enough pressure, we can squeeze the quarks together so close that the strong force between them decreases to a point where their bindings are released. With enough heat, we can make the quarks move fast enough that they can escape the strong force binding them together. This phase transition is what we hope to create and observe.

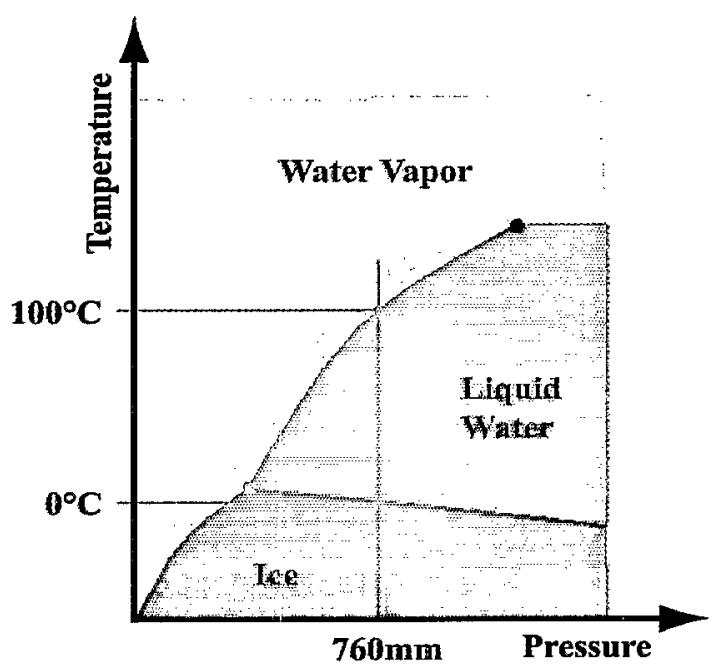

Figure 1a: The phase diagram for water. Three phases of water are plotted as a function of the temperature and pressure of the system. The lines illustrate the familiar freezing and boiling points at normal atmospheric pressure.

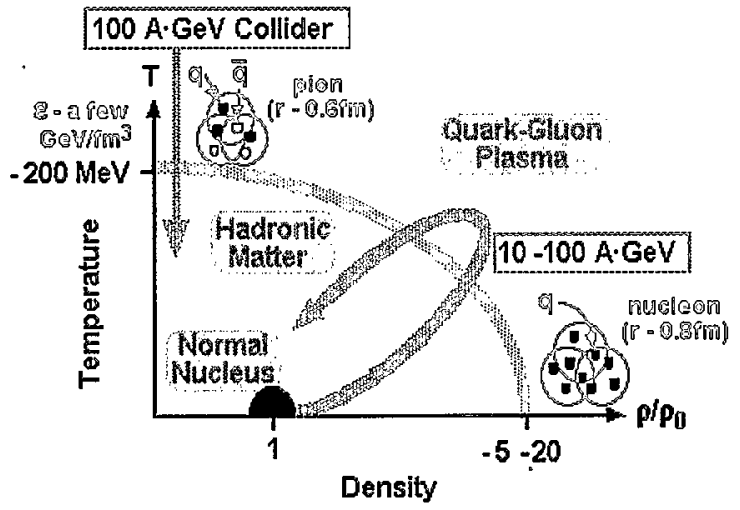

Figure 1b: The phase diagram for nuclear matter with pressure replaced by the nuclear matter density with respect to a normal nucleus. With sufficient temperature and/or pressure, a transition to a QGP could be possible. The green arrows outline the paths we plan to take.

\section{Quark-Gluon Plasma Hide-outs}

There are three possible places where the QGP may be holed up. The first of these is in the early universe, very shortly after the Big Bang. At that time, all of the matter in the universe was compacted into a very small volume at high pressure and temperature where the quarks had not yet grouped together into hadrons. The early universe is thought to have been in a QuarkGluon Plasma phase. As time progressed, the universe expanded and cooled down. At some point (within about $10^{-34}$ seconds after the Big Bang), the universe crossed the nuclear phase transition and the quarks coalesced into hadrons. The universe we live in today took shape $15,000,000,000$ years later. Unfortunately, since the Big Bang happened so long ago, it is extremely difficult for us to use this hide-out to catch the Quark-Gluon Plasma.

Another, more contemporary place we could look for the QGP is in the center of neutron stars, which are the collapsed cores of large stars. A neutron star, like the one in the Crab Nebula, packs the mass of a star into the size of a city. If the center of a neutron star were placed on Brookhaven National Laboratory, its surface would barely reach the north and south shores of Long Island. In the center of a neutron star, conditions of high pressure and high temperature exist, and it is possible that the QGP could be found there. However, neutron stars are very far away and can only be observed with telescopes, making it difficult to probe the interior of the star to determine what lies inside.

Ideally, we would like to build a QGP hide-out in a place of our own choosing so that we could set up a trap to catch the QGP. There is a way to do just that - by using particle accelerators! We plan to bring the QGP to us by choosing the heaviest nucleus that we can work with, typically Gold or Lead, and accelerating that nucleus to relativistic speeds (very close to the speed of light). Then we will slam two of them together. At the point that we produce this collision, we will set up with many surveillance detectors to help us spot the QGP.

\section{Setting Up the QGP Trap}

The particle accelerator approach to pursuing the QGP has been attempted for many years using machines that accelerate one of the nuclei and 
slam it into another nucleus sitting stationary in a laboratory. These types of experiments are called fixed target experiments. One of the first QGP hide-outs set up in this manner was built at the Lawrence Berkeley National Laboratory Bevelac, which accelerated Oxygen to $2 \mathrm{GeV}$ per Nucleon, or $2 \mathrm{GeV} / \mathrm{A}$ for short. Later, more QGP hide-outs were built at the Brookhaven National Laboratory Alternating Gradient Synchrotron (AGS), which accelerated Gold to $11.6 \mathrm{GeV} / \mathrm{A}$, and the CERN Super Proton Synchrotron (SPS), which accelerated Lead to $160 \mathrm{GeV} / \mathrm{A}$. Unfortunately, intensive observation of these hide-outs has produced no absolutely definitive evidence that points to a sighting of the QGP. Despite some close calls, we have not yet been able to bring the QGP in for questioning.

It is possible that these previous hide-outs were not inviting enough to the QGP. So a more QGP-friendly hide-out has been built at the Brookhaven National Laboratory Relativistic Heavy lon Collider, or RHIC for short. RHIC uses one of the previous accelerators, the AGS, as a pre-accelerator for its nuclei. The result is that RHIC can collide nuclei as heavy as Gold at $100 \mathrm{GeV} / \mathrm{A}$. It's like turning the knob up to 11 !

In order to better compare results from the various accelerators (specifically the Bevelac, AGS, SPS, and RHIC), we will use a variable called $s^{1 / 2}$, which represents the amount of energy that is available for particle production in a collision. This is also referred to as the centerof-mass energy. In a fixed target collision of identical nuclei, $s^{1 / 2}$ is given by the equation: $s^{1 / 2}=2 M E+2 M^{2}$, where $M$ is the sum of the mass of both particles and $E$ is the energy of the accelerated nucleus. At the Bevelac, $\mathrm{s}^{1 / 2}=2.5$ $\mathrm{GeV}$. At the AGS, $\mathrm{s}^{1 / 2}=4.9 \mathrm{GeV}$. At the SPS, $\mathrm{s}^{1 / 2}$ increases to $17 \mathrm{GeV}$. The advantage of a collider like RHIC is that all of the energy in the collision is available for particle production, which is not true for fixed target experiments. At a collider, $s^{I / 2}=2\left(E_{l} E_{2}+p_{1} p_{2}\right)+2 M^{2}$, where $E_{l}$ and $E_{2}$ are the energies of each nucleus, $p_{l}$ and $p_{2}$ are their momenta, and $M$ is the sum of their mass. Therefore, RHIC's collisions in the Summer of 2000, which collided $65 \mathrm{GeV} / \mathrm{A}$ Gold, has $s^{1 / 2}=130 \mathrm{GeV}$. RHIC's collisions in the Summer of 2001 collide $100 \mathrm{GeV} / \mathrm{A}$ Gold, or $s^{1 / 2}=200 \mathrm{GeV}$ !

The RHIC accelerator complex consists of many different accelerators. A beam of nuclei is created and handed off from one accelerator to the next. The process begins with some ions (ions are atoms stripped of some of their electrons, thus giving them an electrical charge). The ions are accelerated first in a small tandem accelerator up to about $30 \mathrm{MeV}$. The ions are then transferred into the AGS, where they are further accelerated to relativistic speeds at about $11 \mathrm{GeV}$. The ions are then split into two ion beams and injected into RHIC. The two beams counter-rotate separately around the collider. One beam travels clockwise and the other counter-clockwise. To produce the collisions, the beams are crossed at six points around the collider ring. Currently, surveillance equipment (the experiments) has been placed at four of these beam intersection points.

The four experiments at RHIC are designed to have specific surveillance strengths while simultaneously complementing each other in many observations. There are two large experiments named PHENIX and STAR, and two smaller experiments named BRAHMS and PHOBOS. The PHENIX experiment, which stands for Pioneering High Energy Nuclear Interaction eXperiment, is designed to specifically measure electrons, muons, and photons, which are good probes of the interior of the collision. The STAR experiment, which stands for Solenoidal Tracker At RHIC, is designed specifically to measure as many of the products of the collision as possible using a primary detector that acts much like a large 3-D digital camera. The BRAHMS experiment, which stands for Broad RAnge Hadron Magnetic Spectrometers at RHIC and was named after the famous composer, is designed to look at the region of the collision along the collision axis. The PHOBOS experiment, named after a moon of Mars, is the smallest of the experiments and is designed to quickly measure many properties of the collision. There are many agents involved in the hunt for the QGP. There are over 1000 physicists from all over the globe participating in the four experiments.

\section{The QGP: A Master of Disguise and Deception}

With so much effort put into finding the QGP, why haven't we found it yet? The main reason is that it is very tricky and elusive. It likes to disguise itself and hide under cover in a crowd. When the two nuclei collide, there is a lot of 
energy involved and much of that energy is converted into particles as matter-antimatter pairs ( $E=m c^{2}$ in action). In a RHIC collision, it is estimated that over 5000 particles emerge from the center of the collision. If the QGP is formed, this crowd of particles must be sifted through to find it.

In June, 2000, the RHIC collider was turned on at $\mathrm{s}^{1 / 2}=130 \mathrm{GeV}$, and all four experiments began their surveillance continuing through September 2000. Millions of collisions, also referred to as events, were recorded. After a break to install many improvements to the collider and the experiments, RHIC was restarted at $s^{1 / 2}=200$ $\mathrm{GeV}$ in July 2001. Figure 2 shows photographs of single collisions as seen by STAR, PHENIX, PHOBOS, and BRAHMS showing thousands of particles.

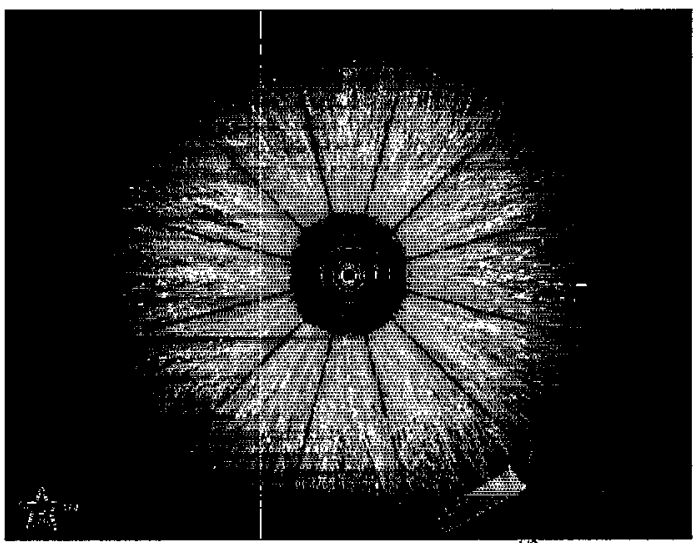

Figure 2a: A Au+Au RHIC collision at $s^{1 / 2}=200 \mathrm{GeV}$ recorded by the STAR experiment. Each line represents a particle. There are thousands of particles seen here.

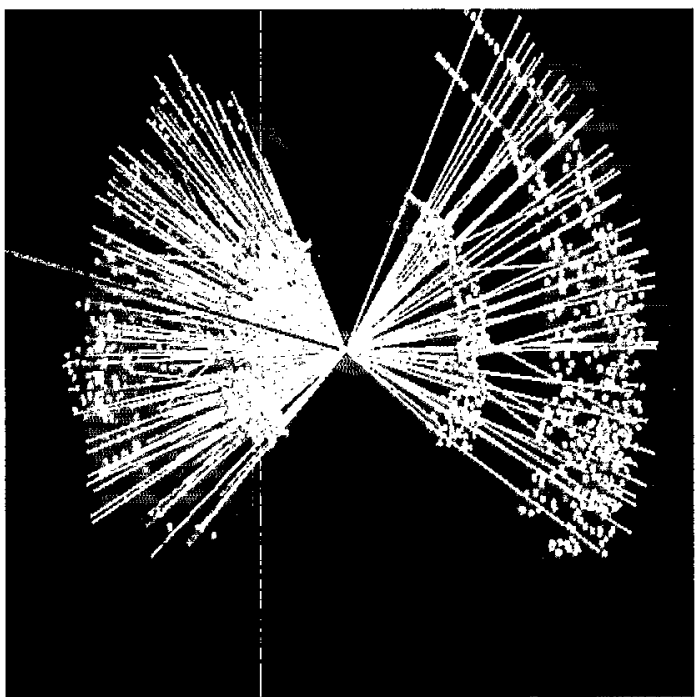

Figure 2b: A Au+Au RHIC collision at $s^{1 / 2}=200 \mathrm{GeV}$ recorded by the PHENIX experiment. Each line and dot represents a particle.

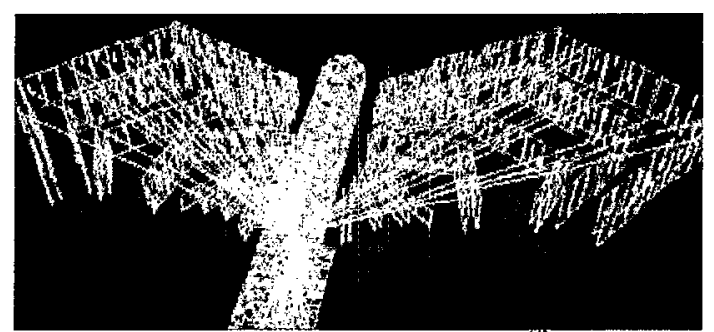

Figure 2c: A Au+Au RHIC collision at $s^{1 / 2}=200 \mathrm{GeV}$ recorded by the PHOBOS experiment. Each line and dot represents a particle.

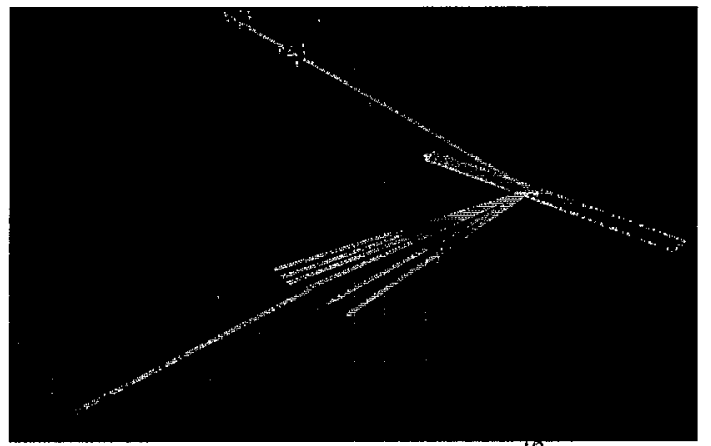

Figure 2d: $\mathrm{A} \mathrm{Au}+\mathrm{Au}$ RHIC collision at $\mathrm{s}^{\mathrm{T} / 2}=200 \mathrm{GeV}$ recorded by the BRAHMS experiment. Each line represents a particle.

\section{RHIC's First Results: The Pursuit of Summer 2000}

Now we will move onto a briefing on some of the attempts to spot the QGP during the Summer of 2000. Most of the efforts focussed on measurements of the properties of the hide-out that was built to determine if it is the correct environment to lure the QGP out of hiding. Measurements on the shape, violence, temperature, fluidity, size, and strangeness of the hide-out were made. Simultaneously, the hunt for the QGP continued in earnest.

Before we continue, a short briefing on the terminology that is used to describe a nuclear collision is in order. We call a head-on collision a central collision. If the collision was only a grazing one, we call it peripheral. We can define a value called the impact parameter, which is the distance between the center of the two nuclei perpendicular to their direction of travel. A directly head-on collision will have an impact parameter of zero. The impact parameter increases for more peripheral collisions.

Since each nucleus consists of individual nucleons, the nuclear collision can be compared to the sum of the individual nucleon-nucleon 
collisions as a baseline. When a measurement is made, we look for differences to the baseline, which could be caused by the activities of the QGP. In order to perform this type of comparison, it is advantageous to express measurements in terms of the number of nucleon-nucleon collisions that occur, which can be extracted from the simple geometry of the collision of two spheres. [Actually, the spheres would appear to be flattened like pancakes due to the fact that they are travelling relativistically and are thus contracted in the direction of travel.] If you examine the region where the two nuclear spheres overlap in the collision and count the number of nucleons in that region, that number is quoted as the number of participants. Those nucleons outside of this region are called spectators, which generally continue travelling in the original directions of each nucleus. If the collision of two identical nuclei is directly headon (central), every nucleon in both nuclei are participants. If the collision is a grazing one (peripheral), there are only a few participant nucleons. Unfortunately, there is no direct way to count the number of participant nucleons in a collision. However, it is possible to estimate this using other means.

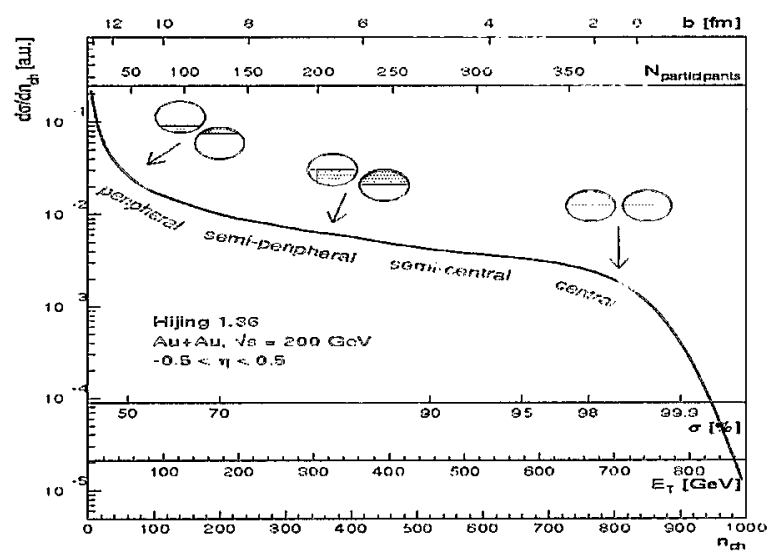

Figure 3: Illustration of the geometry of a nuclear collision. Plotted on the horizontal axis simultaneously are the impact parameter, $b$, the number of participants, the interaction cross section in percent, the total transverse energy, and the total charged particle multiplicity. All of these quantities are related to each other uniquely. The vertical axis is the number of events corresponding to each quantity. The blue curve is the expected shape from a simulation of the collision (called HIJING). Above the curve are schematic pictures of the overlap of the two nuclei at each point.

\section{RHIC Results: The QGP Hide-out Geometry}

A fundamental question that we can ask is: Did we build the hide-out according to our blueprints? Is the hide-out the correct shape? Figure 3 [1] illustrates how we can answer this question. An accelerator shoots nuclei much like a novice pool player. The accelerator can't really aim the two nuclei at each other very well. It can only shoot them in the general direction of each other. Because of this, a collision can be a very rare event, which is only overcome by accelerating very large numbers of nuclei at a time. When a collision occurs, most of the time it will be a peripheral collision, and only very rarely is the aim good enough to produce a central collision.

Knowing this, we can revisit the concept of the collision as consisting of many nucleon-nucleon collisions. Each of these mini-collisions will produce some number of particles. The more mini-collisions that occur, the more particles will be produced overall. Therefore, a central collision will produce many more particles than a peripheral collision. However, there are many more peripheral collisions than central collisions, so if we measure the number of particles produced in each collision and plot that quantity, we would get a shape that looks like the curve in Figure 3. We expect the number of produced particles (also referred to as the multiplicity) to scale with the number of participants uniquely. We can extend this concept to the measurement of the total transverse energy, $E_{t}$, of the produced particles. Here, transverse implies that the energy is moving perpendicular to the collision direction - that is, to the side. Measuring both of these quantities will tell us if we understand the geometry of the collision. Figure 4 [2] shows multiplicity and transverse energy measurements from all four RHIC experiments. All of the experiments measure the same expected shape, implying that the hide-out is solidly built.

[Note: In practice, the experiments use a combination of detectors to determine how central a collision is. This includes a detector that measures the amount of energy remaining directly in the direction of the collision. The shaded area in Figure $4 c$ and the dashed curves in Figure $4 d$ were produced by using this detector for selecting the collisions.] 


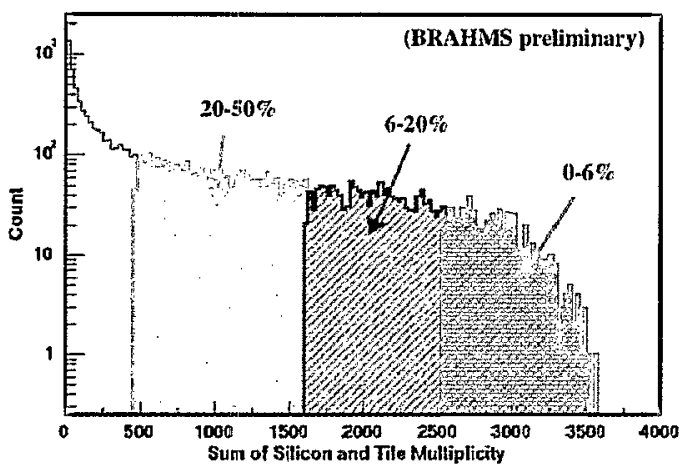

Figure 4a: A measurement of the number of particle "hits", or multiplicity, as measured by the BRAHMS experiment. The percentage of collisions in each region is illustrated by the shading.

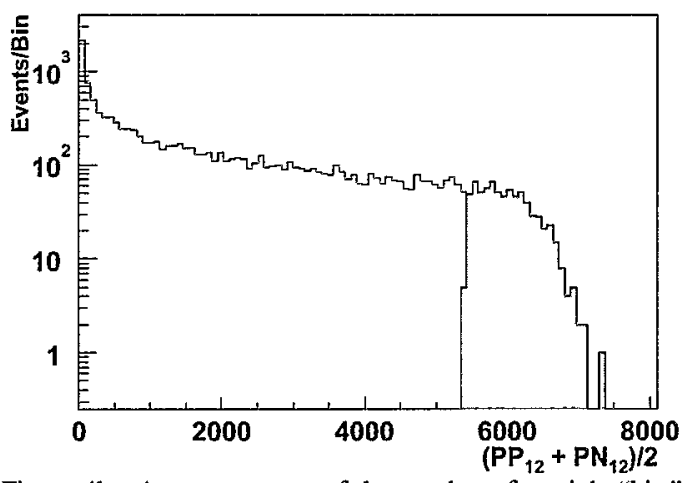

Figure 4b: A measurement of the number of particle "hits", or multiplicity, as measured by the PHOBOS experiment. The shaded region represents the most central collisions.

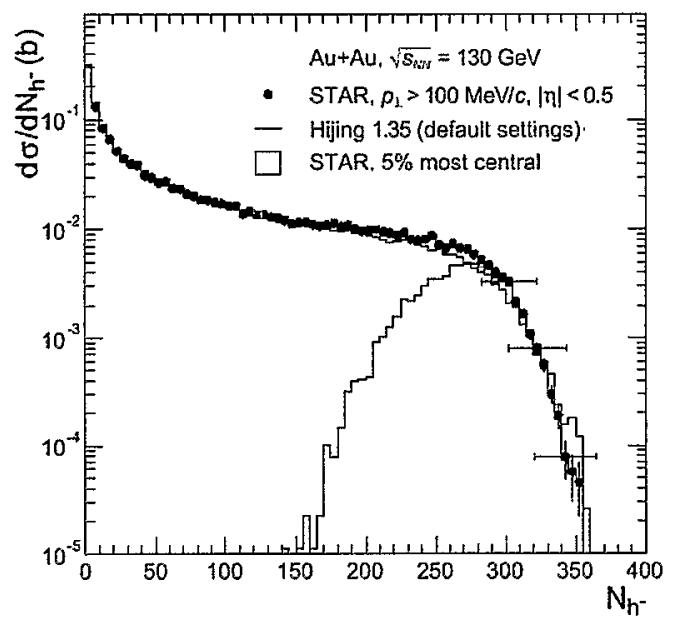

Figure 4c: A measurement of the number of negatively charged particles per collision as measured by the STAR experiment. The shaded region shows the distribution from $5 \%$ of the most central collisions, which have been selected using different detectors. A comparison to the HIJING collision simulation is also shown.

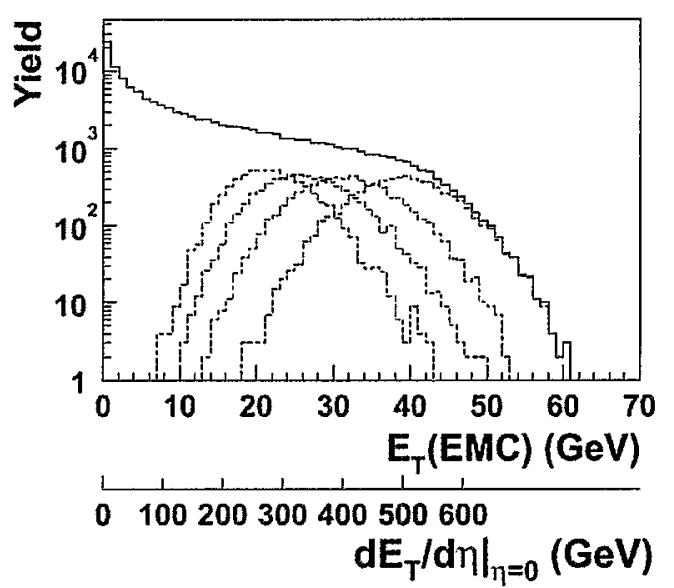

Figure 4d: A measurement of the total transverse energy of particles as measured by the PHENIX experiment. Also shown are the distributions for the most $0-10 \%, 10-20 \%, 20$ $30 \%$, and $30-40 \%$ central collisions.

\section{RHIC Results: The QGP Hide-out Violence}

Have we created an environment that is violent enough for the QGP to show up? To answer this, we will again look at the number of particles that are produced. The more particles that emerge from the collision, the more violent we can consider it to be. Figure 5 [3] shows the number of charged particles as measured by experiments at many different accelerators. When we compare data from more than one accelerator, we will plot the quantity as a function of $\mathrm{s}^{1 / 2}$. Here, the number of charged particles has been normalized to (divided by) the number of participants so that we can directly compare different types of collisions (nucleus-nucleus vs. nucleon-nucleon). Shown on the left are the measurements from the SPS and two measurements by PHOBOS at RHIC (all four experiments agree on the data point at $s^{1 / 2}=130$ $\mathrm{GeV})$. The first thing to note is that the number of particles produced is increasing as the collision energy increases - the system is becoming much more violent. The second thing to note here is that the PHOBOS measurements, especially the $s^{1 / 2}=130 \mathrm{GeV}$ point, is much higher than the nucleon-nucleon measurements, which are plotted separately on the right. This implies that there is some additional process that is producing particles. The system is displaying a collective behavior that is necessary for QGP production. 


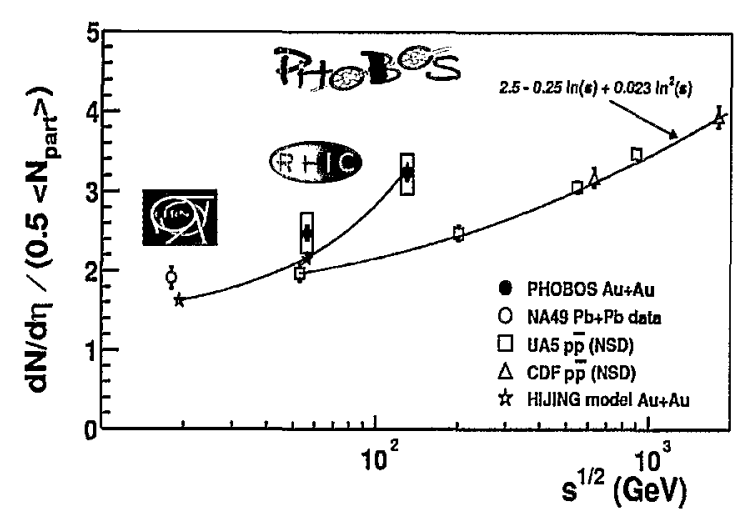

Figure 5: The charged particle multiplicity normalized to the number of participants in the collision as a function of $s^{1 / 2}$ Shown are results from the SPS (NA49) and RHIC (PHOBOS). The curve on the left shows predictions from the HIJING simulation. The points and curve on the right are measurements of the baseline nucleon-nucleon collisions (UA5 and CDF).

The transverse energy produced in the collision can also be examined to help us determine how violent the collision is. Figure 6 [4] shows the total transverse energy produced, again normalized to the number of participants, as measured by PHENIX. Here, peripheral collisions are on the left and central collisions are on the right. The closed circles are the RHIC data while the open circles are the SPS measurements. Again, RHIC shows much more transverse energy production. To quantify this increase, we can use these measurements to estimate the energy density, $\varepsilon_{0}$, in the center of the collision via the Bjorken formula $[5]$ :

$$
\varepsilon_{0} \sim \frac{1}{\pi R^{2} \tau_{0}} \frac{d E_{t}}{d y}
$$

where $\pi R^{2}$ is the effective area of the collision, $\tau_{0}$ is the formation time of the collision $(\sim 1 \mathrm{fm} / \mathrm{c})$, and $\mathrm{dE}_{\mathrm{t}} / d y$ represents the transverse energy directly perpendicular to the collision direction. Plugging these numbers in, we find that at the SPS, the energy density is about 18 times that of normal nuclear matter. At RHIC, it is 29 times that of normal nuclear matter! Having set the world record for collision violence, it appears that the hide-out we have built at RHIC is indeed violent enough for the QGP.

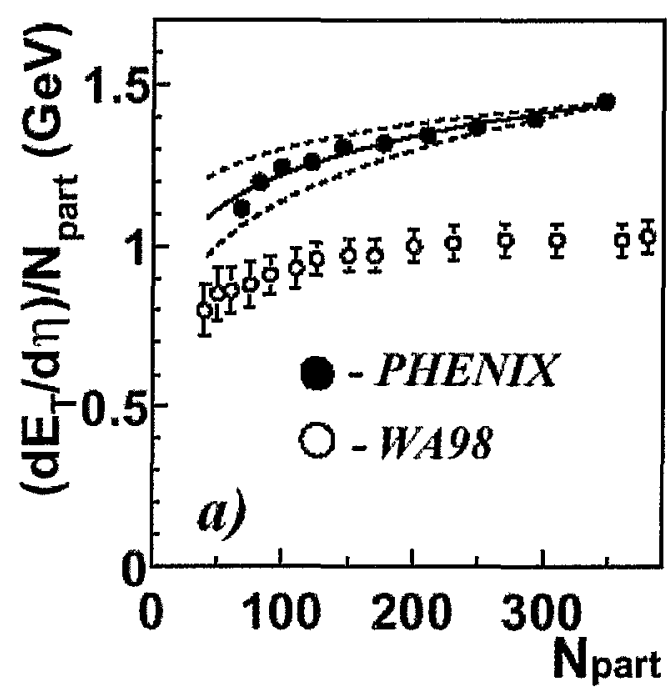

Figure 6: The transverse energy directly perpendicular to the collision direction as measured by the PHENIX experiment (closed circles) normalized to the number of participants. The open circles are the same measurement at the SPS. Here, peripheral collisions are on the left and central collisions are on the right.

\section{RHIC Results: The QGP Hide-out Temperature}

We can set up a thermometer to help take the temperature inside the collision to see if it is hot enough for the QGP. The method applied here is to measure the transverse momentum, $p_{t}$, of particles coming from the collision. To determine the temperature, we assume that the volume in the center of the collision behaves like a gas. In a gas, there are molecules that travel around inside of its volume. If the volume is in thermal equilibrium (that is, it has a uniform temperature), the velocity distribution of the molecules in the gas is described by a MaxwellBoltzmann distribution:

$$
f(v) d v=4 \pi\left(\frac{m}{2 k_{B} T}\right)^{3 / 2} v^{2} \exp \left(-\frac{m v^{2}}{2 k_{B} T}\right) d v
$$

where $m$ is the mass of the particles, $v$ is the velocity, $k_{B}$ is called the Boltzmann constant, and $T$ is the temperature. Since momentum is related to velocity $(p=m v)$, we can use momentum measurements to take the temperature of the system. However, there are particles of different types and different masses being produced, so we can introduce a variable that will allow us to compare all of these particles. This variable is the transverse mass, 


$$
m_{t}=\sqrt{p_{t}^{2}+m_{0}^{2}}
$$

where $m_{0}$ is the rest mass of the particle. When expressed in transverse mass, the MaxwellBoltzmann distribution can be expressed as an exponential distribution, for example:

$$
\frac{1}{m_{t}} \frac{d N}{d m_{t}}=e^{-m_{t} / T}
$$

An experiment can measure the transverse mass and plot it on a semi-log plot. When this is done, the spectrum becomes a straight line whose slope can be related to a temperature. A low temperature system will contain particles with slow velocities producing a steeply-sloped $m_{t}$ spectrum. A high temperature system will contain more particles with high velocities producing a shallow-sloped $m_{1}$ spectrum. Figure 7 [6] plots preliminary results from STAR showing the transverse mass spectrum for negatively charged pions. The exponential fit to the negative pions is with a temperature of 190 $\mathrm{MeV}$. At low $\mathrm{m}_{\mathrm{t}}$, the data deviate from the fit due to the contribution of other processes such as the decay products of unstable particles. Converting the $190 \mathrm{MeV}$ temperature into degrees shows that the collision is more than 100,000 times the temperature at the center of the sun. The hideout appears to be hot enough for the QGP to feel comfortable.

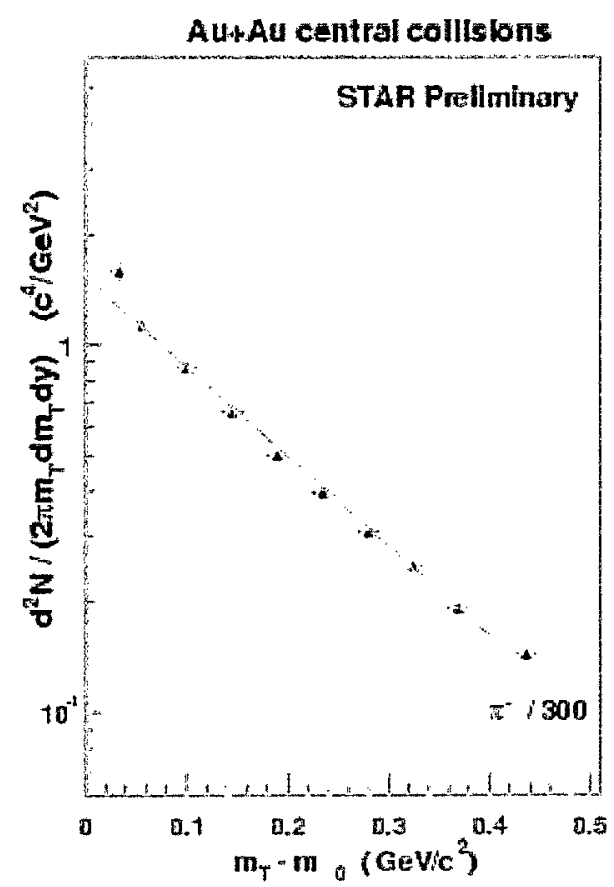

Figure 7: The transverse mass distribution (with the pion rest mass subtracted) for negatively charged pions as measured by the STAR experiment in the 5\% most central collisions. The solid lines are fits performed as described in the text.
There is an experiment performed in many chemistry classes whereby a block of ice is placed in a pot over a lit bunsen burner. In this experiment, the temperature is taken at regular intervals as more energy is pumped into the system. The temperature measurements plateau when the ice melts to water and when the water to turns to steam. Plotting the temperature as a function of time (or as a function of energy pumped into the system) results in the heating curve shown in Figure 8. Figure 9 [7] shows a similar heating curve for nuclear matter plotting the temperature in the nuclear collision as the collision energy increases. Here, it is seen that the temperature appears to exhibit a plateau beginning at the SPS and continuing at RHIC. This is an indication that the hide-out is hot enough for the phase transition to the QGP to occur.

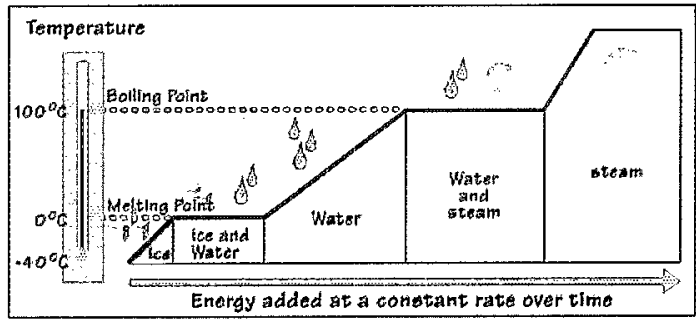

Figure 8: The heating curve for ice to steam showing that the temperature remains constant when the system is undergoing a phase transition.

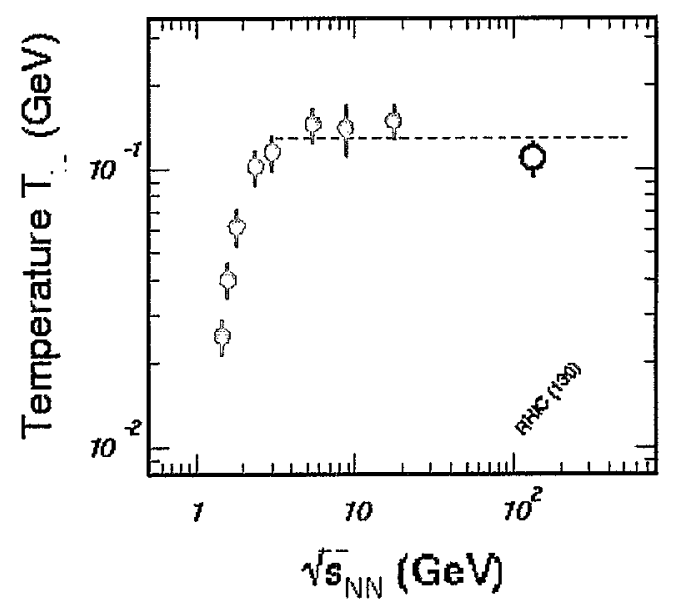

Figure 9: The analogous heating curve measured for nuclear matter from the AGS to RHIC. The temperature appears to exhibit a plateau beginning at SPS energies. 


\section{RHIC Results: The QGP Hide-Out and the Early Universe}

Earlier in the briefing, it was mentioned that one QGP hide-out was the early universe. How well does the RHIC hide-out resemble the early universe? We believe that the ratio of antiprotons to protons in the early universe was very close to one at 0.999999999 . The fact that this value is slightly below one is why most of the universe is made of matter instead of anti-matter today. All four experiments are able to measure this ratio in the RHIC hide-out, and they all agree that it is about 0.6 . This compares to the value below 0.003 at the AGS and below 0.07 at the SPS. Although we have not yet reached the ratio of 1.0, we are rapidly approaching it. The hide-out is starting to look very much like the composition of the universe shortly after the Big Bang.

\section{RHIC Results: The QGP Hide-Out is a Strange Place}

Is the hideout strange enough to make the $Q G P$ comfortable? To determine this, we can measure particles that have strange quarks in them. These particles are very important since all of the strange quarks that are available to make a particle must be produced in the collision - they did not exist at all beforehand. The more strange particles that we see, the more strange quarks must have been produced. Figure 10 [8] shows the ratios of the numbers of various types of particles with strange quarks in them as measured by STAR. Keep in mind that $\Lambda$ particles consist of an up-down-strange quark combination, $\Xi$ particles consist of an upstrange-strange combination, and kaons $(\mathrm{K})$ are made of up-strange combinations. For all cases, when compared to measurements made at the SPS, the amount of anti-matter along with the amount of strange particles produced increases dramatically. The hideout does appear to be strange enough for the QGP to appear.

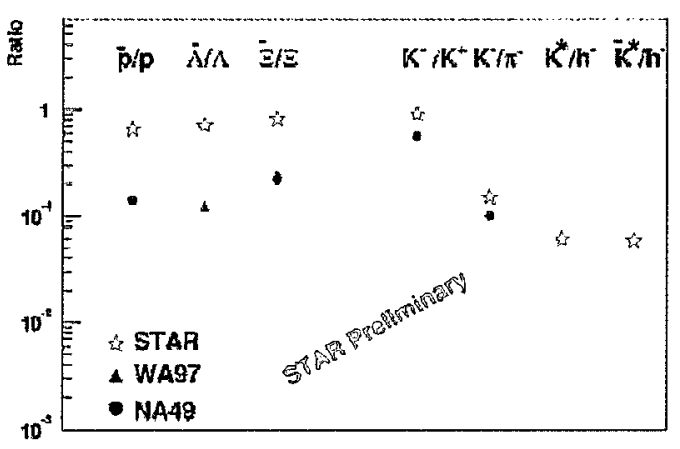

Figure 10: Ratios of various particles as measured by STAR (red stars) and the SPS (blue squares and black diamonds). In all cases, there is more anti-matter and more strange quark production at RHIC than at the SPS.

\section{RHIC Results: Jet Setting}

To summarize what we have learned so far, the preliminary measurements are indicating that the QGP hide-out we have constructed is the correct shape, violent enough, hot enough, strange enough, and is beginning to look like the early universe. But what about the QGP? Has it been spotted yet?

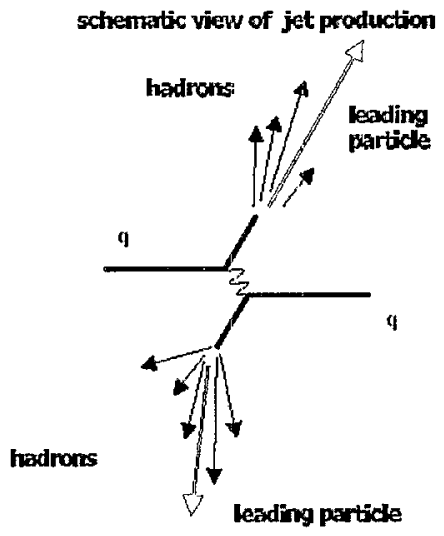

Figure 11: A schematic diagram of the process leading to jet production. The result is several hadrons measured in the detectors aligned back-to-back.

To try and catch a glimpse of the QGP, we can use the fact that it likes to play with jets - it's a real jet-setter. The jets referred to here aren't the 747 variety, however. These jets are produced when an interaction in the collision (such as a particle-antiparticle annihilation) produces two individual quarks that begin to speed away from each other. As mentioned before, quarks are communal particles and do not really like to be by themselves. As they separate, the strong force field between them grows in intensity. Once the force field becomes strong enough, the quarks 
realize that they can use its energy to create their own partners, and so they do. Once they have done that, they cure their loneliness by binding with these partners back into hadrons. As a result, both quarks appear to fragment into several hadrons which continue travelling in the general direction of the original quark. These groups of hadrons are the two jets that end up travelling back-to-back. These hadrons eventually reach the detectors. This process is illustrated schematically in Figure 11.

If the production of jets occurs within a QGP, there is a large amount of matter for the quarks/jets to interact with. As a result, jets that are produced within a QGP will interact strongly with it and tend to lose energy. The QGP appears to slow down the jets. This can be measured by examining the transverse momentum of produced particles. Specifically, we can look at particles with high transverse momentum, where a large fraction of the particles in that region are the result of the jet production process.

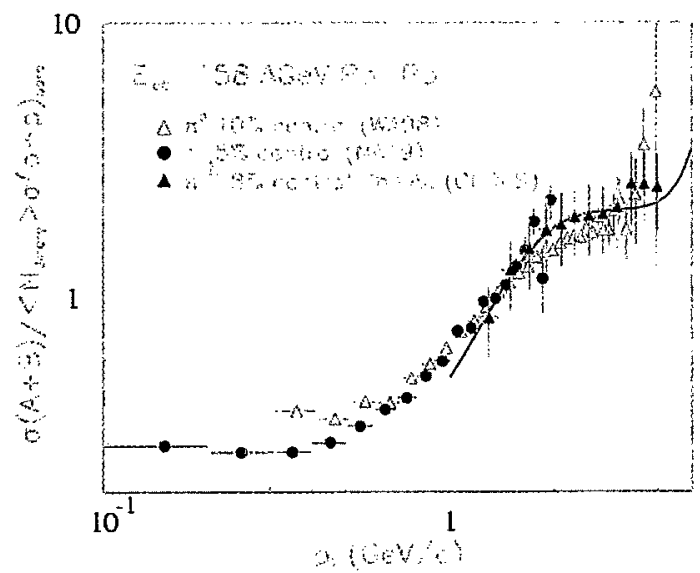

Figure 12: The ratio of the number of charged particles measured in central nucleus-nucleus collisions to that measured in nucleon-nucleon collisions as a function of transverse momentum, as measured by several experiments at the SPS. At high $\mathrm{p}_{\mathrm{t}}$, where jet production processes occur, the ratio is high. The behavior of the data also matches that of the nucleon-nucleus collisions.

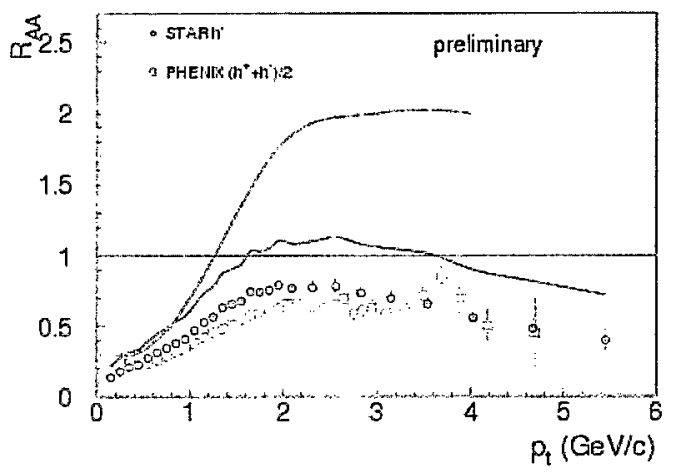

Figure 13: The ratio of the number of charged particles measured in central nucleus-nucleus collisions to that measured in nucleon-nucleon collisions as a function of transverse momentum as measured by PHENIX and STAR at RHIC. At high $\mathrm{p}_{\mathrm{t}}$, where jet processes occur, the ratio is low and decreasing with increasing $\mathrm{p}_{\mathrm{t}}$. This behavior is consistent with jet suppression by a QGP, but it is still too early to know this for sure.

The strategy to catch the QGP with jets is simply to compare the transverse momentum distribution of produced hadrons as measured in individual nucleon-nucleon (and nucleonnucleus) collisions, where there is no jet energy loss expected, to the same distributions in nucleus-nucleus collisions. To do this, we can use nucleon-nucleon measurements taken mostly at Fermilab at various collision energies. Unfortunately, $\mathrm{s}^{1 / 2}$ for these measurements do not match that of RHIC, but it is easy to scale those results to the RHIC energy. After doing that, we simply take the ratio of the nucleus-nucleus spectra to the nucleon-nucleon spectra $\left(\mathrm{R}_{\mathrm{AA}}\right)$. If this ratio is below one at high transverse momentum (typically above $2 \mathrm{GeV} / \mathrm{c}$ ), then there might be an indication of jet energy loss that effectively suppresses the number of these particles. Figure 12 [9] shows this ratio as measured by various experiments at the SPS for central collisions. At high transverse momentum, the ratio tends to continue increasing above one. Also, the trend appears to match that for nucleon-nucleus collisions where no QGP should be present. The SPS data are consistent with a system that does not exhibit the suppression of jets.

However, the ratio as measured by PHENIX and STAR at RHIC behaves in a much different manner, as shown in Figure 13 [10]. At RHIC, the ratio tends to be below one and decreasing as transverse momentum increases. This could be evidence that we have caught a first glimpse of the QGP. However, it is still too early to say for 
sure. We can't haul the QGP in until we have interviewed all of the witnesses. Those witnesses include measurements of nucleon-nucleon and nucleon-nucleus collisions at the RHIC collision energy, which will not be done until later in 2001. Another witness is the difficult analysis of jets which can be associated back-to-back with a photon produced directly in the collision. Identification of these jets provides an unmodified measurement of the initial energy of the jet by measuring the energy of the photon, which does not interact with the QGP. After these witnesses have been cross-examined, this fuzzy glimpse of the QGP should become much clearer.

\section{The Future of RHIC: The Pursuit of Summer 2001}

This concludes the briefing on the results from the first attempt to catch the QGP at RHIC. Although we may have caught a glimpse of our suspect, we will continue our pursuit during the Summer of 2001. This new pursuit will increase the collision energy from $65 \mathrm{GeV} / \mathrm{A}$ to 100 $\mathrm{GeV} / \mathrm{A}$ gold-on-gold collisions. In addition, we will mount the pursuit for four times longer than the one before. This will allow us to increase the statistics on all of the results seen here, gather more data on jet production, and look in detail at the photons (and electrons and muons) that probe the center of the collision so well. In addition, we will take our very own baseline nucleonnucleon (and, if time permits, nucleon-nucleus) collisions for comparison to the nucleus-nucleus collisions. To top it all off, we will be able to accelerate polarized proton beams to help us answer a completely different question: Where does the proton get its spin? You will have to attend a separate briefing to learn more about that topic.

During the summer of 2001, we will be able to bring more powerful surveillance techniques to bear. One example is the measurement of $J / \Psi$ particles. The $\mathrm{J} / \Psi$ consists of a charm quark and an anti-charm quark. Normally, these particles would form with relative ease. However, in the QGP, a phenomenon called color screening could occur. This effect makes it harder for the charm and anti-charm quarks to find each other. This effect would be observed by fewer $J / \Psi$ particles than we expect from nucleon-nucleon collisions. But, how do we identify $\mathrm{J} / \Psi$ particles? These particles are not very stable, and they tend to decay quickly into an electronpositron pair (about $6 \%$ of the time) or a muonantimuon pair (also about $6 \%$ of the time). The PHENIX detector in particular can detect both the electron and muon decays. However, there can be several electrons and muons within a single collision. How do we know which ones come from a $\mathrm{J} / \Psi$ particle? Isolating this particle is done by employing a technique called invariant mass reconstruction. This technique involves looking at every possible pair of electrons (or muons) in the collision. For each pair combination, the kinematics of the decay along with the measured momenta of the particles is used to calculate the mass of a particle, assuming that there was a decay. Most of the time, the calculated mass will be wrong since the chosen pair did not come from a particle that decayed. However, when we choose the correct pair of particles, the calculated mass is exactly correct. If the invariant mass is plotted for every pair, those from the $\mathrm{J} / \Psi$ particle will show a peak at its known mass. The number of pairs in this peak (above the background mass distribution from all of the incorrect pairs) can be counted to determine the $\mathrm{J} / \Psi$ yield. If this yield is smaller than expected, then we may have caught our suspect. This is only one example of the many tools that will be available to us in RHIC's second data taking period.

\section{Summary}

The pursuit of the Quark-Gluon Plasma continues. We believe that we have constructed an environment that the QGP will be comfortable with, and we may have already caught a glimpse of our suspect. We have developed the tools and techniques that will help us bring our suspect in for further questioning. At that time, we look forward to debriefing the suspect and learning much more about what the universe is made of and how it evolved. 


\section{References}

[1] Figure 3 was produced by Thomas Ullrich.

[2] Figure 4a: S.J. Sanders, et al., Proc. $17^{\text {th }}$ Winter Workshop on Nuclear Dynamics, Park City, March 2001. Figure 4b: B.B. Back, et al., Phys. Rev. Lett. 85 (2000) 3100. Figure 4c: C. Adler, et al., nucl-ex/0106004. Figure 4d: K. Adcox, et al., Phys. Rev. Lett. 87 (2001) 052301.

[3] B.B. Back, et al., Phys. Rev. Lett. 85 (2000) 3100.

[4] K. Adcox, et al., Phys. Rev. Lett. 87 (2001) 052301.

[5] J.D. Bjorken, Phys. Rev. D27 (1983) 140.

[6] J. Harris, et al., to be published in Proceedings of the Quark Matter 2001 Conference. Available at http://www.star.bnl.gov/STAR/sds_1/papers/conf_proc.html.

[7] N. Xu, nucl-ex/0104021, to be published in Proceedings of the Quark Matter 2001 Conference.

[8] H. Caines, et al., to be published in Proceedings of the Quark Matter 2001 Conference. Available at http://www.star.bnl.gov/STAR/sds_l/papers/conf_proc.html.

[9] M. Aggarwal, et al., Phys. Rev. Lett. 81 (1998) 4087, G. Agalcishiev, et al., hepex/0003012, and H. Appleshauser, et al., Z. Phys. C46 (1990) 361.

[10] A. Drees, nucl-ex/0105019, to be published in Proceedings of the Quark Matter 2001 Conference.

\section{Additional Information and Further Reading}

Animations illustrating the concepts in this briefing can be found at

http://www.phenix.bnl.gov/WWW/software/luxor/ani/.

Information on the RHIC accelerator complex can be found at http: //Www. bnl.gov/RHIC/.

A tutorial on the concepts of quarks, gluons, and fundamental particles can be found at http://ParticleAdventure.org/.

More about the early universe can be found at http://archive.ncsa.uiuc. edu/Cyberia/Cosmos/CosmicMysteryTour. html.

The slides from this briefing can be found at http://www.phenix.bnl.gov/WWW/publish/mitchell/sambamurti2001/.

Much of the data was presented at the Quark Matter 2001 conference and can be viewed at http://www. rhic.bnl.gov/gm2001/. 